\title{
Levantamento de fitoterápicos manipulados em farmácias magistrais de Dourados-MS
}

\author{
Silvia C. Heredia Vieira, ${ }^{1}$ Soraya Sólon, ${ }^{1}$ Maria do C. Vieira, ${ }^{* 2}$ Néstor A. Heredia Zárate ${ }^{2}$
}

\author{
${ }^{1}$ Curso de Farmácia, Universidade para o Desenvolvimento do Estado e da Região do Pantanal, \\ Rua Alexandre Herculano, 1400, Jardim Veraneio, 79002-080 Campo Grande-MS, Brasil \\ ${ }^{2}$ Faculdade de Ciências Agrárias, Universidade Federal da Grande Dourados, Caixa Postal 533, \\ Rodovia Dourados a Itahum km 12, 79804-970 Dourados-MS, Brasil.
}

\begin{abstract}
RESUMO: Este trabalho teve como objetivo realizar o levantamento dos fitoterápicos manipulados em duas farmácias magistrais de Dourados, enfatizando a constituição das fórmulas (matéria-prima e forma farmacêutica), a possível indicação terapêutica, a origem das drogas vegetais utilizadas na manipulação dos fitocomplexos e a confirmação da existência ou não de artigos científicos que comprovem a utilização das cinco matérias-primas mais manipuladas nas farmácias. Para isso, foram analisados os cadastros ou fichas de pesagem, após a autorização das farmacêuticas responsáveis, do período correspondente a 21 de dezembro de 2006 a 21 de março de 2007. Concluiu-se que: 1 . As matérias-primas mais utilizadas, nas duas farmácias, foram Ginkgo biloba e Hypericum perforatum, coincidentemente, com as maiores demandas e as mesmas concentrações. 2. A credibilidade nos fitoterápicos, por parte da população e dos médicos prescritores, pode ser considerada como baixa, uma vez que apenas $4,1 \%$ das fórmulas manipuladas nas duas farmácias magistrais em estudo corresponderam aos fitoterápicos. 3. Em relação às formas farmacêuticas, houve prevalência de cápsulas gelatinosas, provavelmente, porque a indicação terapêutica das fórmulas tenha sido para uso interno. 4. Dentre as cinco plantas medicinais mais manipuladas, apenas Hoodia gordonii não apresentou comprovação científica e 5 . As plantas medicinais mais utilizadas na manipulação dos fitocomplexos estiveram relacionadas com a perda de peso.
\end{abstract}

Unitermos: Fitoterapia, formas farmacêuticas, manipulação, fitocomplexos.

\begin{abstract}
The aim of this work was to carry out the survey of the phytotherapics manipulated in two magistral drugstores from Dourados, emphasizing the constitution of formulas (raw materials and pharmaceutical form), the possible therapeutic indication, the origin of the vegetal drugs utilized in manipulation of phytocomplexes and the confirmation of the existence or not of scientific articles demonstrating the use of the five most manipulated raw materials in drugstores. Thus, the registration or files weighing were analyzed, with the approval of responsible pharmacists, from the period of $21^{\text {st }}$ December 2006 to $21^{\text {st }}$ March 2007. It was concluded that: 1 . Raw materials most utilized in both drugstores were Ginkgo biloba and Hypericum perforatum, coincidentally both showed the highest demands and the same concentrations. 2. The credibility of the phytotherapics, by the public and prescribers, can be considered low, since just $4.1 \%$ of the manipulated formulas from both magistral drugstores studied corresponded to the phytotherapics. 3. In relation to the pharmaceutical formulas, there was a prevalence of gelatin capsules, probably because the therapeutic indication of formulas had been designed for internal use. 4. Among the five most manipulated medicinal plants, only Hoodia gordonii did not show scientific proof and 5. The medicinal plants most utilized in the manipulation of phytocomplexes were related to weight loss.
\end{abstract}

Keywords: Phytotherapy, pharmaceutical forms, manipulation, phytocomplexes.

\section{INTRODUÇÃO}

As plantas medicinais foram descobertas pelo homem por meio da procura por alimentos, e desde então, foram utilizadas empiricamente para o tratamento de patologias. Num primeiro momento, os medicamentos à base de plantas, em geral, eram utilizados oralmente na forma de pó, infusão (chá) ou decocto e, via tópica, na forma de preparações à base de água ou óleo para unguentos e cataplasmas (Wagner \& Wisenauer, 2006).

O surgimento do conceito de "natural" em muito contribuiu para o aumento do uso das plantas medicinais nas últimas décadas. Para muitas pessoas, esse conceito significa a "ausência de produtos químicos", que são 
aqueles que podem causar algum dano ou, de outra forma, representam perigo. Assim, produtos naturais passaram a ser sinônimo de produtos saudáveis, seguros e benéficos (Mengue et al., 2001). Além desses fatores, o aumento do uso das plantas medicinais também pode ser explicado pelo avanço ocorrido na área científica que permitiu o desenvolvimento de fitoterápicos confiáveis e eficazes. Porém, ainda faltam estudos científicos que comprovem a utilização segura e eficaz de várias plantas (Ribeiro et al., 2005).

O Brasil possui a maior diversidade vegetal do planeta, aproximadamente 55 mil espécies de plantas superiores (Melo et al., 2007) onde, o bioma Cerrado, constitui uma das maiores floras vegetais, compondo um cenário de exuberante diversidade biológica e influente no arcabouço cultural das populações que nele vivem (Vila Verde et al., 2003). Segundo Garlet \& Irgang (2001), o uso dessas plantas, na manipulação dos fitoterápicos, traria vantagens para o país, como redução da importação de medicamentos promovendo, assim, a auto-suficiência e proporcionando à população medicamentos mais baratos e maior valorização das tradições populares.

Segundo a Anvisa (2004), fitoterápico é: "todo medicamento obtido empregando-se exclusivamente matérias-primas vegetais. É caracterizado pelo conhecimento da eficácia e dos riscos de seu uso, assim como pela reprodutibilidade e constância de sua qualidade. Sua eficácia e segurança é validada através de levantamentos etnofarmacológicos de utilização, documentações tecnocientíficas em publicações ou ensaios clínicos fase 3. Não se considera medicamento fitoterápico aquele que, na sua composição, inclua sustâncias ativas isoladas, de qualquer origem, nem as associações destas com extratos vegetais".

Como consequência do aumento da utilização das plantas medicinais, diversos setores mercadológicos vêm produzindo produtos à base de espécies vegetais, em diversas formas farmacêuticas. Contudo, Nunes et al.(2003) indicam que não há garantia da segurança e eficácia para a grande maioria desses produtos. Neste contexto, o presente trabalho teve como objetivo realizar um levantamento dos fitoterápicos manipulados em duas farmácias magistrais de Dourados-MS, enfatizando a constituição das fórmulas (matéria-prima e forma farmacêutica), a possível indicação terapêutica, a origem das drogas vegetais utilizadas na manipulação dos fitocomplexos e a confirmação da existência ou não de artigos científicos que comprovem a utilização das cinco matérias-primas mais manipuladas nas farmácias.

\section{MATERIAL E MÉTODOS}

O trabalho consistiu no levantamento de todos os fitoterápicos manipulados em duas farmácias magistrais de Dourados-MS mediante a análise dos cadastros ou das fichas de pesagem, do período correspondente a 21 de dezembro de 2006 a 21 de março de 2007. Foram analisados os fitocomplexos, um a um, e os dados foram tabulados, com a provável indicação terapêutica de cada planta dentro do fitocomplexo.

Os dados obtidos foram armazenados, tabulados e organizados em ordem decrescente de manipulação para a elaboração de tabelas. A análise quantitativa dos dados consistiu da distribuição de frequências (Ribeiro Júnior, 2001). Também efetuou-se um levantamento bibliográfico procurando a existência de validação científica das cinco plantas medicinais mais utilizadas, em cada farmácia em estudo.

\section{RESULTADOS E DISCUSSÃO}

O levantamento das fórmulas manipuladas nas farmácias magistrais em estudo ( $\mathrm{F} 1=15.002$ fórmulas e $\mathrm{F} 2=17.809$ fórmulas) mostraram que, coincidentemente, apenas $4,1 \%$ corresponderam aos fitoterápicos, sendo que $83 \%$ foram de produtos elaborados com uma única planta. Percebeu-se assim que, mesmo com o avanço no estudo científico das plantas medicinais (Ribeiro et al., 2005), a credibilidade por parte da população e dos médicos prescritores ainda é baixa.

As matérias primas mais utilizadas na manipulação das fórmulas pela Farmácia 1 foram Hypericum perforatum (31) e Hoodia gordonii (24) na forma de extrato seco, Ginkgo biloba (121) na forma de extrato concentrado e Linum usitatissimum (54) como óleo. Já, na Farmácia 2, Ginkgo biloba (186), Hypericum perforatum (44), Valeriana officinalis (38) e Piper methysticum (33) foram as matérias primas mais utilizadas e a grande maioria apresentou-se na forma de extrato seco. Percebeu-se assim que, das matérias primas mais utilizadas, duas foram iguais (Ginkgo biloba e Hypericum perforatum) nas duas farmácias e, as maiores demandas (F1= 49 e 18 e F2= 81 e 26, respectivamente) (Tabela 1), coincidentemente, foram com as mesmas concentrações $(120$ e $300 \mathrm{mg}$, respectivamente).

Dentre as cinco plantas medicinais mais manipuladas, notou-se que somente a Hoodia gordonii não apresentava garantia científica de uso terapêutico, tanto que, em 16 de fevereiro de 2007, foi publicado no Diário Oficial da União, a determinação da Agência Nacional de Vigilância Sanitária (Anvisa) que proibiu, no país, a manipulação de todos os medicamentos à base do extrato vegetal Hoodia gordonii, originário da África e utilizado como inibidor de fome e sede. As outras plantas medicinais são comprovadamente reconhecidas pela comunidade científica sobre a eficácia e segurança.

Observou-se que foram manipuladas treze formas farmacêuticas, prevalecendo cápsulas gelatinosas ( $\mathrm{F} 1=$ $72,8 \% ; F 2=84,5 \%$ ) (Tabela 2). Essa prevalência devese, provavelmente, à indicação terapêutica das fórmulas, já que, a maioria é utilizada pelos pacientes para uso interno. 
Os valores máximos das frequências mensais de preparação de cada uma das formas farmacêuticas manipuladas foram obtidos em meses diferentes, nas duas farmácias, exceto os de cápsulas gelatinosas ( $\mathrm{F} 1=33,9 \%$ e F2 $=33,2 \%$ ), pomada ( $F 1=50,0 \%$ e $F 2=37,5 \%$ ) e creme $(\mathrm{F} 1=54,5 \%$ e $\mathrm{F} 2=50,0 \%)$, que foram em janeiro e os de loção $(\mathrm{F} 1=50,0 \%$ e $\mathrm{F} 2=62,5 \%)$ que foram em março (Tabela 2).

Considerando as preparações farmacêuticas, percebeu-se que a maior prevalência na Farmácia 1 (Tabela 3 ) foi de extrato seco (345), pó (154), extrato concentrado (121), óleo (89) e tintura mãe (32), sendo esta, encontrada apenas nesta farmácia. Já, na Farmácia 2 (Tabela 4), a prevalência foi de extrato seco (575), pó (293), óleo (52), tintura simples (32) e extrato glicólico (21). Esses resultados têm relação com o fato de que nas farmácias magistrais há diferentes tipos de matérias-primas obtidas a partir de uma mesma planta (pó e produtos extrativos), as quais podem ser utilizadas de acordo com a finalidade do produto final.

Comparando as quantidades totais dos tipos de matérias-primas utilizadas, a Farmácia 2 teve 178 fitoterápicos a mais que a Farmácia 1 (801). Além disso, a Farmácia 2 também teve maior número de matériasprimas utilizadas para a obtenção de pó ou de produtos extrativos, podendo citar como exemplo, Prunus amygdalus, Astragalus membranaceus, Adonis vernalis e Cymbopogon winterianus, que não foram utilizadas na Farmácia 1.

Em relação aos fitocomplexos manipulados, os maiores números de diferentes plantas medicinais utilizadas para a manipulação dos mesmos estiveram relacionadas principalmente com o uso como calmante, moderador de apetite, diurético e hepatoprotetor (Tabela 5). Esses resultados demonstram a tendência no uso de fitoterápicos relacionados com a perda de peso.

Dentre as plantas utilizadas na manipulação das fórmulas, apenas treze foram nativas (Tabela 6). Apesar do grande número estimado de espécies vegetais existentes, apenas uma pequena parcela tem sido pesquisada cientificamente quanto ao seu potencial de produção de fármacos. Estes recursos biológicos, foco de interesse mundial face à sua riqueza genética, vêm sendo alvo de biopirataria e de ações governamentais descoordenadas, que dificultam o uso sustentável deste valioso manancial biológico (Vieira \& Silva, 2002).

Tabela 1. Matéria-prima, frequência total e doses utilizadas em duas farmácias magistrais de Dourados-MS, 2006-2007.

\begin{tabular}{|c|c|c|c|c|c|c|}
\hline \multirow{3}{*}{ Matéria-Prima } & \multicolumn{2}{|c|}{ Frequência total } & \multicolumn{4}{|c|}{ Doses } \\
\hline & \multirow[b]{2}{*}{ F1 } & \multirow[b]{2}{*}{ F2 } & \multicolumn{2}{|c|}{ F1 } & \multicolumn{2}{|c|}{$\mathrm{F} 2$} \\
\hline & & & $\begin{array}{c}\text { Concentração } \\
\text { (em mg) }\end{array}$ & Frequência & $\begin{array}{c}\text { Concentração } \\
\text { (em mg) }\end{array}$ & Frequência \\
\hline \multirow{12}{*}{$\begin{array}{c}\text { Ginkgo biloba } \\
24 \%\end{array}$} & \multirow{12}{*}{121} & \multirow{12}{*}{186} & & & 500 & 1 \\
\hline & & & 240 & 6 & 240 & 6 \\
\hline & & & & & 200 & 1 \\
\hline & & & 180 & 3 & & \\
\hline & & & 160 & 12 & 160 & 9 \\
\hline & & & 140 & 1 & & \\
\hline & & & 120 & 49 & 120 & 81 \\
\hline & & & 100 & 11 & 100 & 6 \\
\hline & & & 80 & 33 & 80 & 76 \\
\hline & & & 60 & 1 & 50 & 1 \\
\hline & & & 40 & 5 & 40 & 4 \\
\hline & & & & & 20 & 1 \\
\hline \multirow{9}{*}{$\begin{array}{l}\text { Hypericum } \\
\text { perforatum }\end{array}$} & \multirow{9}{*}{31} & \multirow{9}{*}{44} & 450 & 1 & 450 & 2 \\
\hline & & & & & 400 & 1 \\
\hline & & & & & 350 & 3 \\
\hline & & & 300 & 18 & 300 & 26 \\
\hline & & & 250 & 6 & 250 & 3 \\
\hline & & & 200 & 3 & 200 & 1 \\
\hline & & & & & 150 & 3 \\
\hline & & & & & 120 & 2 \\
\hline & & & 100 & 3 & 100 & 3 \\
\hline
\end{tabular}


Tabela 2. Formas farmacêuticas e frequências, total e mensal, dos fitoterápicos manipulados em duas farmácias magistrais de Dourados-MS, 2006-2007.

\begin{tabular}{lcccccccccc}
\hline \multirow{2}{*}{$\begin{array}{c}\text { Forma } \\
\text { farmacêutica }\end{array}$} & \multicolumn{2}{c}{ Frequência total } & \multicolumn{7}{c}{ Frequência mensal } \\
\cline { 2 - 10 } & F1 & F2 & F1 & F2 & F1 & F2 & F1 & F2 & F1 & F2 \\
\hline Cápsula gelatinosa & 446 & 620 & 30 & 72 & 151 & 206 & 148 & 186 & 117 & 156 \\
Cápsula oleosa & 70 & 31 & 4 & 2 & 18 & 13 & 38 & 10 & 10 & 6 \\
Loção & 8 & 8 & 1 & - & - & - & 3 & 3 & 4 & 5 \\
Creme & 11 & 18 & 2 & 1 & 6 & 8 & 3 & 7 & - & 2 \\
Pomada & 10 & 5 & - & - & 4 & 3 & 3 & 2 & 3 & - \\
Oleoleto & 2 & 10 & - & - & 1 & 2 & - & 4 & 1 & 4 \\
Solução alcoólica & 65 & 23 & 8 & 3 & 27 & 7 & 8 & 9 & 22 & 4 \\
Gel & 1 & 2 & - & - & - & 1 & - & 1 & 1 & - \\
Sabonete & - & 10 & - & 1 & - & 5 & - & 1 & - & 3 \\
Xampu & - & 2 & - & - & - & 1 & - & 1 & - & - \\
Solução & - & 2 & - & - & - & - & - & 2 & - & - \\
Condicionador & - & 1 & - & - & - & - & - & 1 & - & - \\
Xarope & - & 4 & - & - & - & 1 & - & 2 & - & 1 \\
\hline TOTAL & 613 & 736 & 45 & 79 & 207 & 247 & 203 & 229 & 158 & 181 \\
\hline
\end{tabular}

Tabela 3. Matérias-primas vegetais utilizadas para a manipulação de fitoterápicos em duas farmácias magistrais de Dourados-MS, 2006-2007. Farmácia 1

\begin{tabular}{lll}
\hline Preparações farmacêuticas & Frequência & Matéria prima \\
\hline Pó (droga vegetal) & Valeriana officinalis (21); Baccharis trimera (17); Aesculus hippocastanum (14); Centella asiatica \\
& (14); Fucus vesiculosus (14); Persea americana (12); Rhamnus purshiana (12); Crataegus oxyacantha \\
& (11); Salix alba (10); Podofilina hexandrum (9); Cassia angustifolia (9); Lepidium peruvianum \\
& (4); Psyllium sp. (4); Echinodorus macrophillus (3); Paullinia cupana (3); Curcuma zedoaria (3); \\
& Polygonum sp. (2); Harpagophytum procumbens (2); Hamamelis virginiana (2); Humulus lupulus \\
& (2); Chamomilla recutita (1); Erythroxylum catuaba (1); Equisetum sp. (1); Erythrina mulungu (1); \\
& Pfaffia paniculata (1); Cas'earia sylvestris (1); Solanum paniculatum (1); Passiflora incarnata (1); \\
& Ptycopetalum olacoldes (1); Melissa officinalis (1); Uncaria tomentosa (1)
\end{tabular}

Extrato concentrado

Extrato fluido

Extrato glicólico

Extrato Seco

Tintura Simples

Tintura mãe

32

Óleo$$
\text { (1) }
$$

Ginkgo biloba (121)

Casearia sylvestris (4); Arctium lappa (2); Eucalyptus sp. (2); Passiflora alata (2); Melissa officinalis (2); Valeriana officinalis (2); Gentiana lutea (1); Mikania glomerata (1); Erythrina mulungu (1); Phyllanthus sp. (1); Croton campestris (1)

Calendula officinalis (14); Aesculus hippocastanum (2); Ginkgo biloba (2); Hamamelis virginiana (2); Arnica montana (3); Rosmarinus officinalis (1); Centella asiatica (1); Symphytum officinale (1)

Hypericum perforatum (31); Hoodia gordonii (24); Rhamnus purshiana (20); Passiflora alata (18); Cynara scolymus (17); Garcinia cambodja (15); Piper methysticum (14); Panax ginseng (10); Tanacetum partenum (8); Valeriana officinllis (8); Camellia sinensis (7); Tribullus terrestris (7); Solanum melongena (6); Cassia nomame (6); Cimicifuga racemosa (6); Serenoa repens (3); Uncaria tomentosa (3); Bixa orellana (3); Dioscorea villosa (3); Citrus aurantium (2); Rhamnus purshiana (2); Agnus castus (1); Angelica sinensis (1); Garcinea camboja e Garcinea indica (1); Gymnena silvestre (1)

Scaptotrigona postica (5); Centella asiatica (2); Thuya occidentalis (2); Arnica montana (1); Arctium lapa (1); Peumus boldus (1); Calendula officinalis (1); Ginkgo biloba (1); Valeriana officinallis (1)

Thuya occidentalis (5); Smilax aspera (3); Calendula officinalis (2); Aesculus hippocastanum (2); Ginkgo biloba (2); Hamamelis virginiana (2); Sabal terrulata (2); Persea americana (1); Aesculus hippocastanum (1); Aloe vera (1); Euphorbia entheurodoxa (1); Avena sativa (1); Chamomilla recutita (1); Echinacea angustifolia (1); Maytenus ilicifolia (1); Hypericum perforatum (1); Tabebuia avellanedae (1); Mentha piperita (1); Plantago major (1); Curcuma zedoaria (1); Zingiber aromaticum (1)

Linum usitatissimum (54); Oenothera biennis (14); Cymbopogon nardus (5); Prunus amygdalus (3) Melaleuca lanceolata (3); Allium sativum (2); Helianthus annus (2); Rosa aff. rubiginosa (2); Citrus reticulata (1); Copaifera sp. (1); gérmen de Triticum aestivum (1); semente de Vitis vinifera (1) 
Tabela 4. Matérias-primas vegetais utilizadas para a manipulação de fitoterápicos em duas farmácias magistrais de Dourados-MS, 2006-2007. Farmácia 2

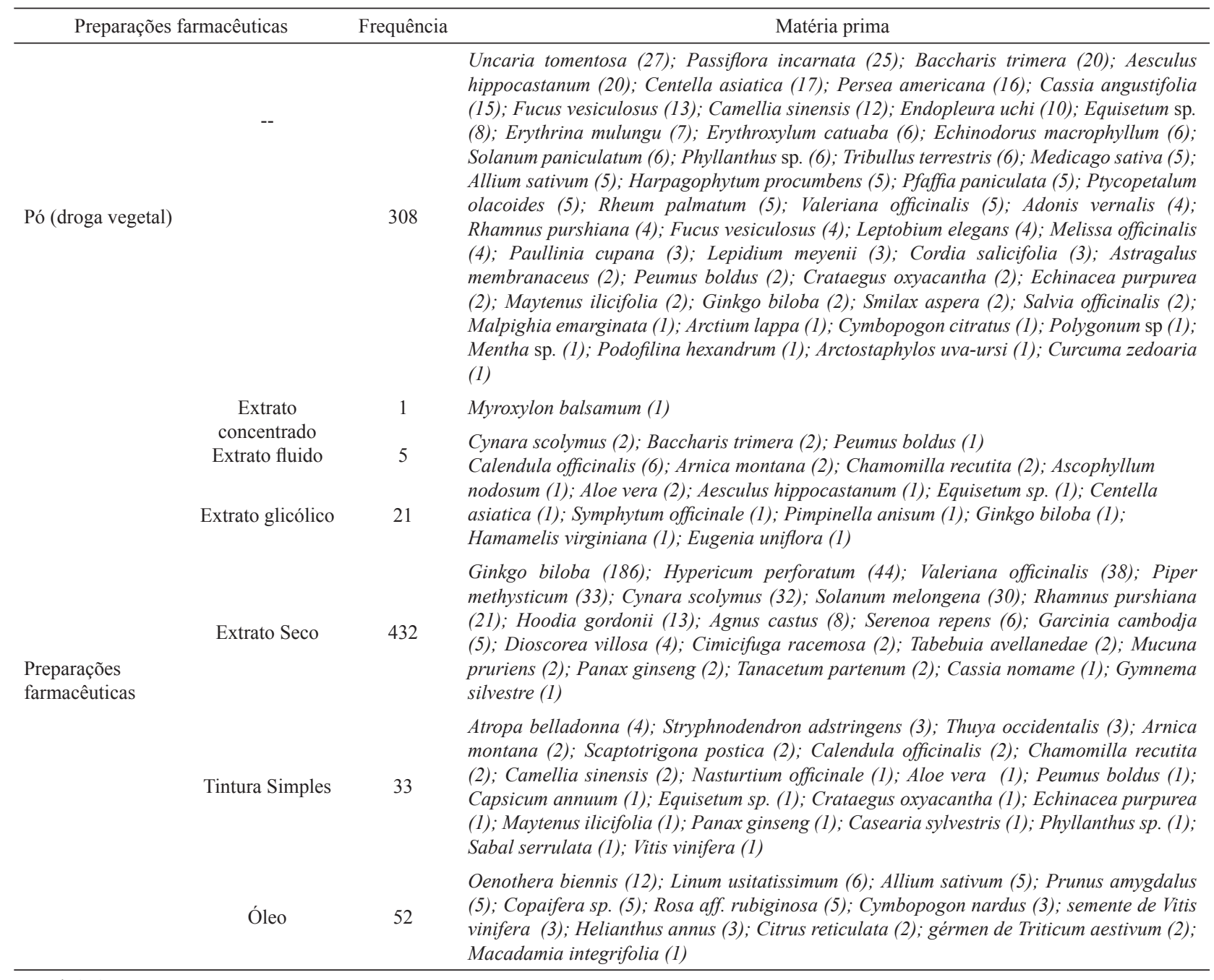

Total: 979

Tabela 5. Propriedades medicinais de matérias-primas utilizadas em duas farmácias magistrais de Dourados-MS, 2006-2007.

\begin{tabular}{ll}
\hline Propriedades medicinais & \multicolumn{1}{c}{ Matéria prima } \\
\hline Afrodisíaco & Erythroxylum catuaba; Ptycopetalum olacoides; Tribullus terrestris \\
Analgésico & Arnica montana; Peumus boldus; Cymbopogon citratus; Maytenus ilicifolia; Salix Alba \\
Antigripal & Echinacea purpurea; Mentha sp.; Bixa orellana; Rosmarinus officinalis \\
Antimicrobiano & Rosmarinus officinalis; Allium sativum; Cymbopogon citratus; Cymbopogon nardus; Maytenus \\
& ilicifolia; Curcuma zedoaria \\
Antioxidante & Camellia sinensis; gérmen de Triticum aestivum; Capsicum annuum; Uncaria tomentosa; Vitis vinifera \\
& semente \\
Antisséptico & Rosmarinus officinalis; Arctium lappa; Scaptotrigona postica; Calendula officinalis; Chamomilla \\
& recutita, Maytenus ilicifolia; Hypericum perforatum; Curcuma zedoaria \\
Antitumoral & Podofilina hexandrum; Maytenus ilicifolia \\
Artrite & Polygonum sp. \\
Cálculos renais & Phyllanthus sp. \\
Calmante & Cymbopogon citratus; Crataegus oxyacantha; Humulus lupulus; Hypericum perforatum; Piper \\
& methysticum; Passiflora alata; Melissa officinalis; Erythrina mulungu; Passiflora alata; Valeriana \\
officinalis & Adonis vernalis
\end{tabular}




\begin{tabular}{|c|c|}
\hline Cicatrizante & $\begin{array}{l}\text { Rosmarinus oficinalis; Arnica montana; Aloe vera; Calendula officinalis; Chamomilla recutita; } \\
\text { Centella asiatica; Hypericum perforatum }\end{array}$ \\
\hline Contra diabetes & Gymnena silvestre \\
\hline Controle de mioma & Endopleura uchi \\
\hline Distúrbios de memória & Panax ginseng; Ginkgo biloba; Paullinia cupana \\
\hline Distúrbios menstruais & Agnus castus; Chamomilla recutita; Salvia officinalis; Dioscorea villosa \\
\hline Diurético & $\begin{array}{l}\text { Persea americana; Rosmarinus oficinalis; Medicago sativa; Arctium lappa; Cymbopogon citratus; } \\
\text { Equisetum sp.; Centella asiatica; Echinodorus macrophyllum; Polygonum sp.; Solanum paniculatum; } \\
\text { Maytenus ilicifolia; Cordia salicifolia; Smilax áspera }\end{array}$ \\
\hline Estimulante digestivo & Rosmarinus oficinalis; Peumus boldus; Baccharis trimera \\
\hline Hepato-protector & $\begin{array}{l}\text { Cynara scolymus; Rosmarinus oficinalis; Allium sativum; Baccharis trimera; Harpagophytum } \\
\text { procumbens; Solanum paniculatum; Erythrina mulungu; Salvia officinalis; Curcuma zedoaria }\end{array}$ \\
\hline Hidratante & Prunus amygdalus; Rosa aff. Rubiginosa \\
\hline Hipoglicemiante & Syzygium cumini; Mucuna pruriens \\
\hline Infecções respiratórias & Echinacea purpurea; Mentha sp. \\
\hline Infecções urinárias & Polygonum sp.; Uncaria tomentosa; Arctostaphylos uva-ursi \\
\hline Laxante & Echinodorus macrophyllum; Rhamnus purshiana; Rheum palmatum; Cassia angustifólia \\
\hline Menopausa ("fogachos") & Hypericum perforatum; Salvia officinalis \\
\hline Melhora circulação & Centella asiatica Hamamelis virginiana; Uncaria tomentosa; Curcuma zedoaria \\
\hline $\begin{array}{l}\text { Moderador de apetite } \\
\text { (emagrecimento). }\end{array}$ & $\begin{array}{l}\text { Baccharis trimera; Citrus aurantium; Garcinia cambodja; Hoodia gordonii; Cordia salicifolia; Fucus } \\
\text { vesiculosus. }\end{array}$ \\
\hline Prisão de ventre & Rhamnus purshiana; Rheum palmatum \\
\hline Redução do colesterol & Solanum melongena; Bixa orellana \\
\hline Revitalizar cabelos & Macadamia integrifólia \\
\hline Úlcera & Calendula officinalis; Maytenus ilicifolia; Solanum paniculatum \\
\hline
\end{tabular}

Fontes: Corrêa (1926-1978), Font Quer (1993), Lorenzi \& Matos (2002). Ferro (2006)

Tabela 6. Origem das plantas (nativas e exóticas) utilizadas em duas farmácias magistrais de Dourados-MS, $2006-2007$.

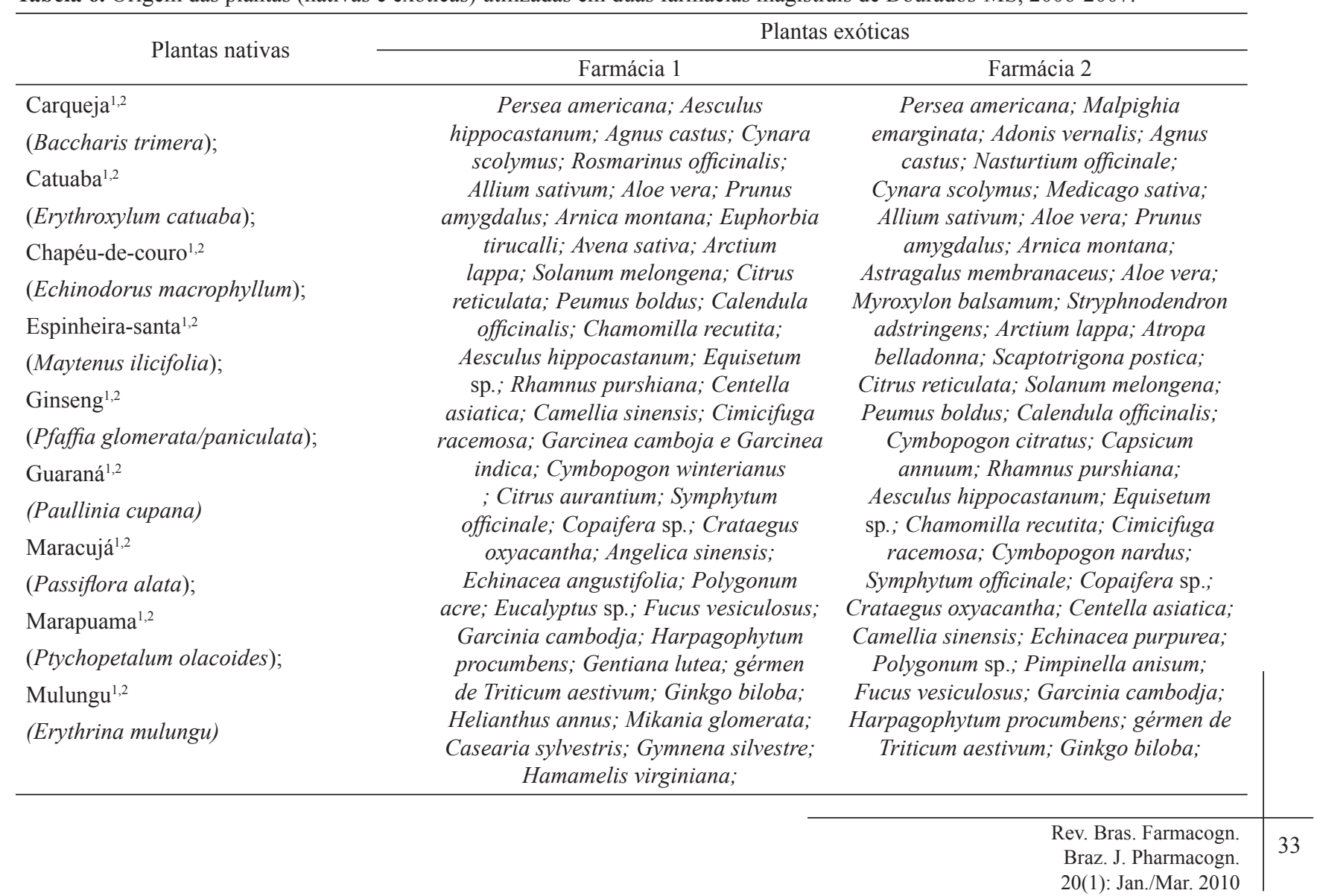




\begin{tabular}{|c|c|c|}
\hline $\begin{array}{l}\text { Quebra-pedra }{ }^{1,2} \\
\text { (Phyllantus } \mathrm{sp}) \\
\text { Unha-de-gato }^{1,2} \\
\text { (Uncaria tomentosa); } \\
\text { Uxi-amarelo }^{2} \\
(\text { Endopleura uchi) } \\
12\end{array}$ & $\begin{array}{c}\text { Hoodia gordonii; Humulus lupulus; } \\
\text { Hypericum perforatum; Tabebuia } \\
\text { avellanedae; Solanum paniculatum; } \\
\text { Piper methysticum; Linum usitatissimum; } \\
\text { Lepidium meyenii; Melaleuca } \\
\text { lanceolata; Melissa officinalis; Mentha } \\
\text { piperita; Panax ginseng; Podofilina } \\
\text { hexandrum; Oenothera biennis; } \\
\text { Psyllium; Rhamnus purshiana; Rosa aff. } \\
\text { rubiginosa; Sabal terrulata; Salix alba; } \\
\text { Smilax aspera; Serenoa repens; semente } \\
\text { de Vitis vinifera; Cassia angustifolia; } \\
\text { Tanacetum partenum; Plantago major; } \\
\text { Thuya occidentalis; Tribullus terrestris; } \\
\text { Uncaria tomentosa; : Bixa orellana; } \\
\text { Valeriana officinalis; Croton campestris; } \\
\text { Dioscorea villosa; Curcuma zedoaria; } \\
\text { Zingiber aromaticum. } 80\end{array}$ & $\begin{array}{l}\text { Helianthus annus; Casearia sylvestris; } \\
\text { Gymnema silvestre; Hamamelis } \\
\text { virginiana; Hoodia gordoni; Mentha } \\
\text { sp.; Hypericum perforatum; Tabebuia } \\
\text { avellanedae; Solanum paniculatum; } \\
\text { Piper methysticum; Leptobium elegans; } \\
\text { Linum usitatissimum; Lepidium meyenii; } \\
\text { Macadamia integrifolia; Melissa } \\
\text { officinalis; Mucuna pruriens; Panax } \\
\text { ginseng; Passiflora incarnata; Eugenia } \\
\text { uniflora; Oenothera biennis; Podofilina } \\
\text { hexandrum; Cordia salicifolia; Rosa } \\
\text { aff. rubiginosa; Rheum palmatum; } \\
\text { Sabal serrulata; Smilax aspera; } \\
\text { Salvia officinalis; Serenoa repens; } \\
\text { semente de Vitis vinifera; Cassia } \\
\text { angustifolia; Tanacetum partenum; } \\
\text { Thuya occidentalis; Tribullus terrestris; } \\
\text { Arctostaphylos uva-ursi; Valeriana } \\
\text { officinalis; Dioscorea villosa; Curcuma } \\
\text { zedoaria. } 82\end{array}$ \\
\hline
\end{tabular}

${ }^{1}$ Plantas nativas utilizadas na farmácia $1 .{ }^{2}$ Plantas nativas utilizadas na farmácia 2.

\section{CONCLUSÕES}

Nas condições em que foi desenvolvido o trabalho concluiu-se que as matérias-primas mais utilizadas, nas duas farmácias, foram de plantas medicinais exóticas, com prevalência de Ginkgo biloba e Hypericum perforatum, coincidentemente, com as maiores demandas e as mesmas concentrações; a credibilidade nos fitoterápicos, por parte da população e dos médicos prescritores, pode ser considerada baixa; em relação às formas farmacêuticas, houve prevalência de cápsulas gelatinosas, provavelmente, porque a indicação terapêutica das fórmulas tenha sido para uso interno; dentre as cinco plantas medicinais mais manipuladas, apenas Hoodia gordonii não apresentou comprovação científica; e, as plantas medicinais mais utilizadas na manipulação dos fitocomplexos estiveram relacionadas com a perda de peso.

\section{REFERÊNCIAS}

Anvisa 2004. Resolução RDC nº 48, de 16 de março de 2004. Disponível em: http://e-legis.bvs.br/leisref/public/ showAct.php?id=10230. Acessada em julho de 2007.

Corrêa MP 1926-1952. Dicionário das plantas úteis do Brasil e das exóticas cultivadas. Rio de Janeiro: Imprensa Nacional, 1926-1952 (v. I a III), 1969-1978 (v. IV a VI) (6v. ilustrados).

Ferro D 2006. Legislação de Fitoterapia. In: Ferro D. Fitoterapia: conceitos clínicos. São Paulo: Atheneu, p. 83-114.

Font Quer P 1993. Plantas medicinales: el dioscórides renovado. 3.v. Espanha: Labor S.A.

Garlet TMB, Irgang BE 2001. Plantas medicinais utilizadas na medicina popular por mulheres trabalhadoras rurais de Cruz Alta, Rio Grande do Sul, Brasil. Rev Bras Pl Med 4: 9-18.

Lorenzi H, Matos FJA 2002. Plantas medicinais no Brasil: nativas e exóticas. Nova Odessa: Plantarum.

Melo JG, Martins JDGR, Amorim ELC, Albuquerque UP 2007. Qualidade de produtos a base de plantas medicinais comercializados no Brasil: castanha-da-índia (Aesculus hippocastanum L.), capim-limão (Cymbopogon citratus (DC.) Stapf) e centela (Centella asiatica (L.) Urban). Acta Bot Bras 21: 27-36.

Mengue SS, Mentz LA, Schenkel EP 2001 Uso de plantas medicinais na gravidez. Rev Bras Farmacogn 11: 21-35.

Nunes GP, Silva MF, Resende UM, Siqueira JM. 2003. Plantas Medicinais comercializadas por raizeiros no Centro de Campo Grande, Mato Grosso do Sul. Rev Bras Farmacogn 13: 83-92.

Ribeiro AQ, Leite JPV, Dantas-Barros AM 2005. Perfil de utilização de fitoterápicos em farmácias comunitárias de Belo Horizonte sob a influência da legislação nacional. Rev Bras Farmacogn 15: 65-70.

Ribeiro Júnior JI 2001. Análises estatísticas no SAEG. Viçosa: UFV.

Vieira RF, Silva SR 2002. Estratégias para a conservação e manejo de recursos genéticos de plantas medicinais e aromáticas: resultados da $1^{a}$ Reunião Técnica. Brasília: Embrapa/ Ibama/ CNPq

Vila Verde GM, Paula JR, Caneiro DM 2003. Levantamento etnobotânico das plantas medicinais do cerrado utilizadas pela população de Mossâmedes (GO). Rev Bras Farmacogn 13: 64-66.

Wagner H, Wisenauer M 2006. Fitoterapia: Fitofármacos, farmacologia e aplicações clínicas. 2 ed. São Paulo: Pharmabooks. 